\title{
Ein weiterer Beitrag über die steigenden Gesundheitskosten aus anderer Perspektive
}

\author{
R. Kurmann
}

Ach ja, waren das früher noch Zeiten, als ein Albert Schweitzer karitativ ohne entsprechende Entlöhnung wirkte. Damals dominierte im Gesundheitssektor vorwiegend noch eine auf Hilfe und Nonprofit ausgerichtete Ethik. Zur Genesung und Lebensrettung von Menschen wurde für Ehre, Kost und Logis oder für ein minimes Entgelt jederzeit verfügbar rund um die Uhr gearbeitet. Als Beispiel seien hier die Ordensschwestern genannt. Helfen und Mindern von Leid standen im Vordergrund. Gesundheit sollte ideell allen zur Verfügung stehen. Entsprechend flossen später auch aus staatlichen Stellen, privaten Subventionen und Spenden entsprechend Gelder. Aus dem Solidaritätsgedanken heraus entstanden die Krankenkassen, die obligatorische Unfallversicherung und Invalidenversicherung. Die gesundheitliche Versorgung wurde immer mehr zu einer Selbstverständlichkeit, um deren Kosten sich niemand sorgen sollte.

Springen wir doch ein Jahrhundert weiter. Zwischenzeitlich ist das Gesundheitswesen zu einem riesigen Wirtschaftszweig mutiert, an dem viele verdienen und verdienen wollen. Zeiten und Mentalität haben sich radikal geändert. Allein bei Schnelldurchsicht der Schweizer Börse zählen wir 18 kotierte Schweizer Unternehmen, die im Gesundheitssektor tätig sind (Roche, Novartis, Galenica, Serono, Actelion, Berna Biotech, Comet, Cytos Biotech, IVF Hartmann, Nobel Biocare, Straumann, Phonak, Centerpuls, Synthes Stratec, Card Guard, Unilabs, Disetronic). Dazu kommen noch viele internationale Firmen, deren Papiere an der Schweizer Börse gehandelt werden, da die Schweiz zu den profitablen Absatzmärkten zählt, zumal ja unser Land auch als geschütztes hochmargiges Hochpreisparadies weltweit geschätzt wird. Diese im Gesundheitsmarkt tätigen Firmen ordnen sich wie alle anderen Firmen und Wirtschaftszweige gewinnorientierten, auf Wachstum, ShareholderValue und Profit ausgerichteten ökonomischen Strategien unter. Allein diese Unternehmen beschäftigen Millionen von Mitarbeitern, die alle Arbeit und ein Salär erhalten, für die Teppichetagen reicht es sogar immer noch zu Salären, von denen ein Arzt nur träumen kann, und fetten Boni in Millionenhöhe. Die Nachfrage am
Markt kann durch ein breites Angebot jederzeit befriedigt werden. Durch Werbung und Marketingstrategien werden mit Schlagworten wie Lifestyle, Fitness, Wellness, Komfort, Gesundheitsbewusstsein die Märkte nicht nur befriedigt, sondern mehr noch systematisch und gewinnorientiert ausgeweitet, um eben Wachstum und Shareholder-Value zu genügen. Werden doch auch immer mehr Lebensbereiche und Krisen in unserem Dasein entdeckt und gefördert, wo es im Sinne der Marktausweitung immer noch etwas zu optimieren und die Lebensqualität zu steigern gibt bzw. Gewinne erwirtschaftet werden können. Teure Neuerungen lassen in der Regel auch nicht lange auf sich warten, die bisherigen Ausrüstungen werden dann einfach medial und marketinggerecht als nicht mehr zeitgemäss, belastend und als dringend zu ersetzen deklariert. Ersatzteile für die älteren Geräte sind auch entsprechend schnell nicht mehr lieferbar. Wirtschaftsberater, Ökonomen und Marketingstrategen drängen da ebenfalls massenweise in diesen Markt, um für gutes Geld uns allen noch mehr Effizienz, Wachstum und Gewinn in Aussicht zu stellen. Dass die Wirtschaft hier nur Kundenbedürfnisse und die Nachfrage abdeckt, darf angesichts der obig geschilderten Realitäten also nur der kleinere Teil der Wahrheit sein, vielmehr müssen die Unternehmen stets steigende Gewinne und Profite erwirtschaften.

Sollten da die Terminologien nicht einmal endlich zeitgemäss im Sinne des zunehmenden Wirtschaftsneoliberalismus geändert und ersetzt werden?

Das ehemals vorwiegend idealistisch gefärbte Gesundheitswesen ist längst zum gewinnorientierten, profitablen Gesundheitsmarkt mutiert, Patienten und Leistungserbringer sind zu Kunden und Konsumenten der Wirtschaft avanciert. Die Albert-Schweitzer-Zeit ist angesichts solcher Tatsachen wohl endgültig vorbei, natürlich bestätigen auch hier Ausnahmen die Regel.

Mittlerweile hat dieser Markt derartige Ausmasse erreicht, dass die bisherigen, an den früheren Zeitgeist angelehnten Finanzierungsmodelle in arge Nöte kommen bzw. nicht mehr genügen. $\mathrm{Zu}$ lange hat sich in den Köpfen die Mentalität gehalten, dass Gesundheit und Medizin nichts 
oder nur wenig kosten dürfen und irgendwie subventioniert werden. Es mutet nun geradezu abenteuerlich an, dass in diesem Wirtschaftsgefüge der Arzt allein die entscheidende Stellschraube zur Kostensenkung im Gesundheitsmarkt sein soll. Glauben das unsere Politiker wohl im Ernst? Zumal jetzt wissenschaftliche Untersuchungen [1] belegen, dass zwischen Ärztedichte und Gesundheitskosten kein signifikanter Zusammenhang besteht, wie das gerne von Krankenkassen und Politikern immer wieder medial kolportiert und argumentativ quasi als Beweis ins Feld geführt wird. Eher drängt sich der Verdacht auf, dass man der Ärzteschaft am bequemsten und einfachsten den Schwarzen Peter unterschieben kann. Bekanntlich nimmt der Arzt zur Kenntnis und schweigt nobel, schlimmstenfalls ist ausser Murren in den eigenen Reihen und Fäusten in den Hosentaschen aus dieser heterogenen und in sich unstimmigen Berufsgruppe ohne politische Lobby eh kein nennenswerter Widerstand $\mathrm{zu}$ erwarten. Es ist nicht schwer zu erraten, wer hier der lachende Dritte ist.

\section{Literatur}

1 Horisberger B, Schmid O. Ärztestopp als Kostenbremse - Warum es Zeit ist, umzudenken. Schweiz Ärztezeitung 2004;85(17):887-94. 